
[0212-7199(2003) 20: 2; pp 91-100]
ANALES DE MEDICINA INTERNA
Copyright $\odot 2003$ ARAN EDICIONES, S.L.

AN. MED. INTERNA (Madrid) Vol. 20, N. ${ }^{\circ} 2$, pp. $91-100,2003$

\title{
Tratamiento actual de la tuberculosis
}

\author{
R. GARCÍA RAMOS, F.L. LADO LADO', V. TÚNEZ BASTIDA², M.L. PÉREZ DEL \\ MOLINO BERNAL ${ }^{3}$, A. CABARCOS ORTIZ DE BARRÓN ${ }^{1}$ \\ Servicio de Farmacia y ${ }^{\prime}$ Medicina Interna. Departamento de Medicina. ${ }^{2}$ Unidad de \\ Tuberculosis. Servicio de Medicina Preventiva. ${ }^{3}$ Servicio de Microbiología. Complejo \\ Hospitalario Universitario de Santiago. Santiago de Compostela. La Coruña
}

\author{
CURRENT TREATMENT OF TUBERCULOSIS
}

\begin{abstract}
RESUMEN
La tuberculosis (TB) es una enfermedad conocida desde la antigüedad y a pesar de los grandes avances en la medicina continúa siendo un problema grave de salud a nivel mundial. En este trabajo se realiza una revisión actualizada del tratamiento farmacológico de la tuberculosis teniendo en cuenta los principios generales de actuación de los medicamentos antituberculosos tanto de primera como de segunda línea. Se recogen los esquemas terapéuticos tanto de la enfermedad tuberculosa como de la tuberculosis latente. Se presentan las reacciones adversas y las interacciones resumidas en tablas así como actuaciones a tener en cuenta para minimizarlas. Se describen las actuaciones para mejorar la adherencia al tratamiento y el control del cumplimiento terapéutico, y el tratamiento aconsejado en situaciones especiales como inmunodeficiencias, embarazo, insuficiencia hepática y renal, y la problemática de las multirresistencias. La perspectiva de tener a corto plazo nuevos tratamientos más eficaces para combatir esta enfermedad y que rebajen la duración de éstos no es muy prometedora.
\end{abstract}

PALABRAS CLAVE:Tuberculosis. Tratamiento. Reacciones adversas. Interacciones.

\begin{abstract}
Tuberculosis $(T B)$ is a growing national and international health concern today. A revision of pharmacological treatment of TB is done in this issue. Regimens for TB disease and latent TB treatment are descri bed. Common adverse reactions and drug interactions of first and second-line antituberculosis drug are summarized in tables. Management strategies to improve treatment adherence and TB control, treat special situations like immunodeficiences, pregnancy, hepatic and renal impairment and multidrug resistant $T B$ are presented. In a next future the prospects for using more effective and shorter new treatments to fight against this disease are not very promising.
\end{abstract}

KEY WORDS: Tuberculosis. Treatment. Antituberculosis drug adverse reactions. Interactions.

García Ramos R, Lado Lado FL, Túnez Bastida V, Pérez del Molino Bernal ML, Cabarcos Ortiz de Barrón A. Tratamiento actual de la tuber culosis. An Med Interna (Madrid) 2003; 20: 91-100.

\section{INTRODUCCIÓN}

Aunque la tuberculosis (TB) es una enfermedad conocida desde antiguo, su tratamiento farmacológico es relativamente reciente. Los progresos en la terapéutica se han sucedido de forma lenta pero continua desde que Waksman descubrió la estreptomicina en 1944. A partir de ese momento en el tratamiento de la TB existen 3 fechas decisivas: es descubrimiento de la isoniacida en 1952, la introducción de la rifampicina en 1967 y cuando la pirazinamida se incorpora al tratamiento como medicamento de primera línea al final la década de los 70, lo que permitió acortar el tratamiento a 6 meses. Sin embargo, la tuberculosis continúa siendo en importante pro- blema de salud en todo el mundo, puesto que, pese a tener estas combinaciones efectivas de medicamentos, al ser tan prolongado el tratamiento se produce en una elevada proporción un mal cumplimiento de éste por parte de los enfermos. Esto, y además de la negligencia en el control de la infección y enfermedad, según la la Organización Mundial de la Salud (OMS), está ocasionando la aparición de multirresistencias en todo el mundo principalmente en países de la antigua Unión Soviética y Asia como consecuencia del desmantelamiento de los servicios de salud pública y la crisis económica (1).

En nuestro medio la incidencia de la TB se mantiene en cifras muy altas que no están de acuerdo con nuestro nivel de desarrollo económico y sanitario (2-6). Los factores más

Trabajo aceptado: 14 de mayo de 2002

Correspondencia: F. L. Lado Lado.Servicio de Medicina Interna. Hospital Clínico Universitario. C/ A Choupana, s/n. 15706 Santiago de Compostela. La Coruña 
importantes de la falta de control de la enfermedad son por una parte las incorrecciones en la práctica médica en cuanto al manejo de la enfermedad (retrasos diagnósticos que condicionan un aumento del riesgo de transmisión de la enfermedad e importantes incorrecciones en cuanto al manejo de las pautas terapéuticas instauradas), y por otra parte, el abandono del tratamiento por parte del paciente, que se ha mostrado como el problema más serio para el control de la TB y el mayor obstáculo para la eliminación de la enfermedad. La acción sanitaria más importante para acelerar el declive de la TB y conseguir el control de la misma es el tratamiento precoz de todos los enfermos y la correcta cumplimentación de la pauta terapéutica instaurada. Para garantizar esta última se debería intentar conseguir la instauración de un porcentaje creciente de Tratamientos Directamente Observados (TDO), tal y como recomienda la OMS, que incluso preconiza su universalización.

\section{PRINCIPIOS GENERALES DEL TRATAMIENTO}

Una terapia farmacológica eficaz reduce rápidamente la población de bacilos viables y con ello el riesgo de transmisión. El tratamiento de los casos de esputo positivo debe realizarse con la máxima premura (7). La hospitalización debe evitarse y sólo será necesaria en casos muy graves (particularmente como resultado de una complicación grave, como hemoptisis copiosa o pioneumotórax) o que se encuentran postrados en cama con una paraparesia grave. Casi todos los demás casos se pueden tratar satisfactoriamente en el domicilio haciendo un seguimiento del cumplimiento del tratamiento. Los corticosteroides no se recomiendan para el tratamiento ordinario de la TB, pero en medio hospitalario pueden ser útiles pautas breves en los casos de pericarditis, derrame pleural, meningitis tuberculosa, tuberculosis miliar aguda con disnea y excepcionalmente en casos de reacciones severas de hipersensibilidad a los medicamentos antituberculosos $(8,9)$.

Es imprescindible conocer los principios bacteriológicos que rigen la quimioterapia de la TB para evitar errores en el planteamiento terapéutico, ya que un tratamiento incorrecto podría conducir al fracaso y al desarrollo de resistencias a los fármacos antibacilares. Los fármacos que se emplean en la pauta corta de 6 meses son la isoniacida $(\mathrm{H})$, rifampicina $(\mathrm{R})$, pirazinamida $(\mathrm{Z})$, etambutol $(\mathrm{E})$ y estreptomicina $(\mathrm{S})$. Son considerados fármacos de primera línea en función de su eficacia y tolerabilidad. Sin embargo, el tratamiento de pacientes tuberculosos plantea diversas dificultades: los bacilos son sólo sensibles a los fármacos bactericidas cuando están activos metabólicamente y en replicación; además, durante periodos indefinidos permanecen subpoblaciones de bacilos que sólo se activan de modo transitorio durante lapsos muy cortos y por último, puede haber mutantes farmacorresistentes incluso en poblaciones de bacilos que nunca han estado previamente expuestas a los antibióticos.

Así pues, las características fundamentales en las que se basa la quimioterapia corta son su elevado poder bactericida contra bacilos metabólicamente activos y una actividad esterilizante contra los bacilos que persisten en estado semilatente, así como su capacidad para prevenir las resistencias mientras dure el tratamiento. Consiste en el uso simultáneo de varios fármacos $(\mathrm{R}, \mathrm{H}$ y $\mathrm{Z})$ para que se produzca la eliminación de las distintas poblaciones bacilares y en una duración suficien- te (6 meses) para evitar la aparición de recidivas. La $\mathrm{R}$ es el único medicamento bactericida contra las tres poblaciones antes mencionadas. La H, S y otros aminoglucósidos son bacteridas contra los microorganismos que se localizan en el medio extracelular. La $\mathrm{H}$ también resulta bactericida contra los bacilos intracelulares. La Z sólo es bactericida contra aquellos intracelulares y actúa muy bien en medio ácido en la fase inflamatoria de la enfermedad (primera fase o dos primeros meses). De los medicamentos de segunda línea o elección, que se utilizan para las presentaciones de tuberculosis resistentes a los de primera o como alternativa en situaciones clínicas aisladas, las fluorquinolonas (FQ), capreomicina $(\mathrm{Cm})$, protionamida $(\mathrm{Pt})$, etionamida $(\mathrm{Et})$, kanamicina $(\mathrm{Km})$ y rifabutina tienen actividad bactericida. Otros medicamentos de segunda línea como: ácido paraaminosalicílico (PAS), cicloserina (Cs), clofazimina (Cf), y macrólidos tienen acción bacteriostática.

La eficacia de los medicamentos antituberculosos depende de la dosis, que debe realizarse de acuerdo con el peso y la edad. Siempre que sea posible se han de administrar en ayunas y en una sola toma y no debe ingerirse alimento hasta que no hayan pasado 15-30 minutos. En las tablas I y II se recogen las dosis habituales de los fármacos de primera y segunda línea de tratamiento respectivamente (10-14).

\section{TABLA I}

DOSIS RECO M ENDADAS PARA EL TRATAM IENTO INICIAL DE LA TUBERCULOSIS

\begin{tabular}{lcc}
\hline Medicamento & Dosis diaria & $\begin{array}{c}\text { Dosis } 2 \text { veces/ semana } \\
\text { (intermitente) }\end{array}$ \\
\hline Isoniacida (H) & $\begin{array}{c}5 \mathrm{mg} / \mathrm{kg} \\
\text { D. máx. } 300 \mathrm{mg}\end{array}$ & $\begin{array}{c}15 \mathrm{mg} / \mathrm{kg} \\
\text { D. máx: } 900 \mathrm{mg}\end{array}$
\end{tabular}

Rifampicina ( $R$ )

$\begin{array}{cc}10 \mathrm{mg} / \mathrm{kg} & 10 \mathrm{mg} / \mathrm{kg} \\ \text { D. máx. } 600 \mathrm{mg} & \text { D. máx: } 600 \mathrm{mg}\end{array}$

Pirazinamida (Z)

$\begin{array}{ll}30 \mathrm{mg} / \mathrm{kg} & 50-70 \mathrm{mg} / \mathrm{kg} \\ \text { D. máx. } 2 \mathrm{~g} & \text { D. máx: } 4 \mathrm{~g}\end{array}$

Etambutol (E)

$15-25 \mathrm{mg} / \mathrm{kg}^{*}$ $50 \mathrm{mg} / \mathrm{kg}$

$\begin{array}{ccc}\text { Estreptomicina (S) } & 15 \mathrm{mg} / \mathrm{kg} & 25-30 \mathrm{mg} / \mathrm{kg} \\ & \text { D. máx. } 1 \mathrm{~g}^{* *} & \text { D. máx. 1,5 g** }\end{array}$

*25 mg/kg 2 primeros meses seguido de $15 \mathrm{mg} / \mathrm{kg}$. **120 g máximo por ciclo tratamiento

\section{ESQUEMAS TERAPÉUTICOS}

Continúan siendo válidos los criterios consensuados en 1991 (10) para el tratamiento de enfermos iniciales, considerando como tales los que no han recibido ningún tipo de tratamiento antituberculoso o lo han hecho por menos de un mes: 2 meses con $\mathrm{H}, \mathrm{R}$ y Z seguido de 4 meses con $\mathrm{H}$ más R. Siempre que sea posible se hará uso de especialidades farmacéuticas que combinen los 2 ó 3 principales fármacos, pues evitan monoterapias y favorecen el cumplimiento asegurándose que cubran las dosis recomendadas (Tabla I). Las presentaciones actuales de estas asociaciones son: Rifater ${ }^{\circledR}$ (Aventis) que 
TABLA II

DOSIS RECO M ENDADAS DE M EDICAM ENTOS DE SEGUNDA LÍNEA DE TRATAM IENTO DE LA TUBERCULO SIS

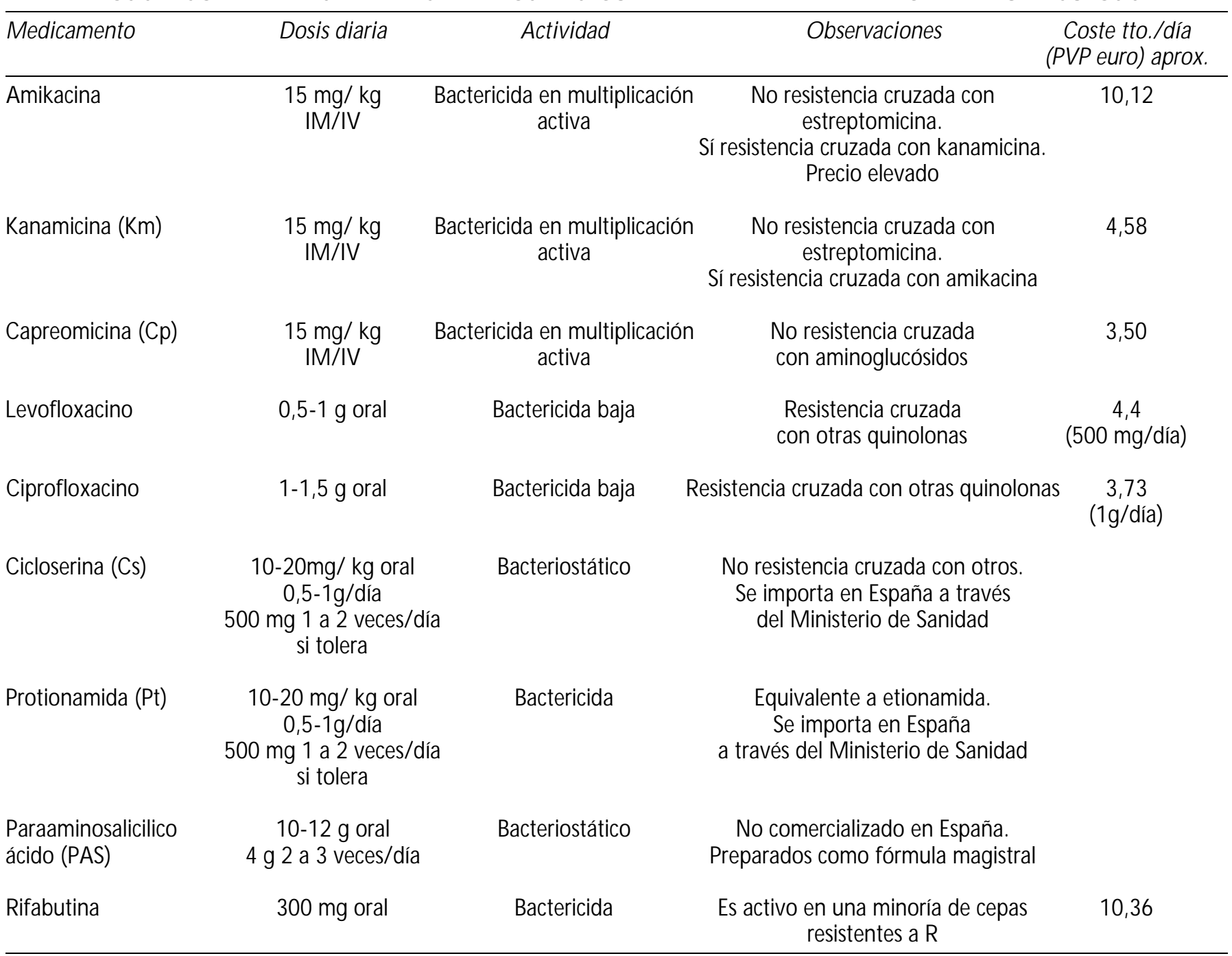

contiene $120 \mathrm{mg}$ de R, $50 \mathrm{mg}$ de $\mathrm{H}$ y $300 \mathrm{mg}$ de Z (dosis diaria: 4 comprimidos para enfermos con peso menor a $40 \mathrm{~kg}, 5$ entre 41 y $60 \mathrm{~kg}$, y 6 más de $61 \mathrm{~kg}$ ); Rifinah ${ }^{\circledR}$ (Aventis) y Rimactazid $®$ (Novartis) que contienen $300 \mathrm{mg}$ de R y $150 \mathrm{mg}$ de $\mathrm{H}$ (dosis diaria: 2 comprimidos).

Estudios realizados en España han detectado resistencias primarias a $\mathrm{H}$ variables y más recientemente de sólo el 2,9\% en población reclusa (4). Esta prevalencia sugiere, al ser inferior al $4 \%$, que la pauta de 3 fármacos es suficiente. No obstante, es aconsejable investigar esta prevalencia en diversas áreas geográficas para determinar la situación real de la resistencia y considerar la introducción un cuarto fármaco, E o S. Estos dos fármacos deben formar parte de la pauta inicial en poblaciones con tasas superiores de resistencia primaria (inmigrantes de Europa del Este, Latinoamérica, África y Asia).

Las distintas pautas de tratamiento de TB inicial no multirresistente presentan una fase inicial "intensiva" de dos meses con al menos 3 fármacos con una administración diaria, segui- da una fase mantenimiento con dos fármacos en la que la administración puede ser diaria o 2 veces a la semana (tratamiento intermitente). Esta última opción es igual de efectiva que la diaria (15), pero siempre debe de realizarse bajo estricta observación (TDO).

El esquema de elección para el tratamiento de pacientes inmunocompetentes es 2HRZ/4HR con las siguientes modificaciones: a) si la incidencia de resistencia primaria a $\mathrm{H}$ en el área es mayor del $4 \%$ se debe adicionar un cuarto medicamento los 2 primeros meses: $\mathrm{E}$ o $\mathrm{S}, \mathrm{b}$ ) si el paciente no debe tomar $\mathrm{Z}$ (artritis gotosa, insuficiencia hepática o renal severa, intolerancia) la pauta recomendada es $2 \mathrm{HRE} / 7 \mathrm{HR}$, c) si hay que evitar $\mathrm{H}$ el régimen propuesto es $2 \mathrm{EZR} / 10 \mathrm{ER}$ y d) si no se puede emplear R: 2HZE/4HE. En el caso de inmunodepresión se aconseja ampliar la fase de mantenimiento 3 meses más.

El paciente debe de ser siempre remitido a una unidad especializada ante un fallo terapéutico (fracaso, recidiva o abandono) o si tiene antecedente de contacto con pacientes con cepas multirresistentes y el tratamiento debe basarse en los estudios 
de sensibilidad. Si se trata de un paciente con antecedente de tuberculosis (fallo terapéutico), el retratamiento se determinará a partir de la pauta previa y su cumplimiento.

\section{TRATAMIENTO DE TUBERCULOSIS LATENTE}

En la actualidad, se prefiere utilizar la denominación tratamiento de TB latente mejor que tratamiento preventivo o quimioprofilaxis, con el fin de promover un mayor entendimiento del concepto tanto en los pacientes como en los profesionales sanitarios. Este tratamiento se considera una medida esencial para el control epidemiológico de la TB (16).

Hasta hace poco tiempo, la $\mathrm{H}$ era el único fármaco con evidencias clínicas de eficacia en el tratamiento de la TB latente. Durante la última década se han completado diversos estudios clínicos de tratamiento de TB latente en pacientes con infección por el virus de la inmunodeficiencia humana (VIH) que han establecido la posibilidad de tratamientos cortos como alternativa a la pauta clásica de $\mathrm{H}$ durante 6-12 meses $(16,17)$. El esquema de tratamiento habitual promovido por el Consenso $(4,10)$ es de $\mathrm{H}$ durante 6 meses y durante 9-12 meses en los infectados por VIH, en los que puede utilizarse también $\mathrm{R}$ más $\mathrm{Z}$ durante 2 meses. La segunda pauta se reservará para pacientes que pueden retrasar 2 meses el inicio del tratamiento antirretroviral o que no reciben tratamiento triple con inhibidores de proteasa (18). Si se utiliza esta pauta se recomiendan controles a las 2,4,6 semanas de enzimas hepáticas y bilirrubina, ya que se han notificado casos de mortalidad por hepatitis severa (19). Otra pauta ensayada en VIH es 3 meses de R más H (20). Las administraciones pueden ser dos veces a la semana (tratamiento intermitente), en tal caso se recomienda hacerlo en régimen de TDO.

Aunque no existen evidencias clínicas al respecto, en los casos en los que no es recomendable usar $\mathrm{R}$ (pacientes con infección por el VIH en tratamiento con inhibidores de la proteasa), se puede sustituir por rifabutina. La manera más segura de realizar este tipo de tratamiento sería monitorizando los valores de los inhibidores de la proteasa.

$\mathrm{El}$ régimen terapéutico de $\mathrm{R}$ con $\mathrm{Z}$ durante 2 meses no ha sido suficientemente evaluado en sujetos no infectados por el $\mathrm{VIH}$, aunque parece razonable que sea igual o más eficaz que en estos pacientes (16).

En la actualidad la pauta clásica de 6 meses de H se está cuestionando. En 1999 la American Thoracic Society (ATS) y los Centers for Disease Control and Prevention (CDC) establecieron nuevas recomendaciones para las pruebas de tuberculinas y tratamiento de la TB latente en base a los resultados de ensayos clínicos que analizaron. Consideran como recomendación "preferida" en pacientes inmunocompetentes un tratamiento con H durante 9 meses y como alternativa "aceptable" $\mathrm{H}$ durante 6 meses, $\mathrm{R}$ durante 4 meses diariamente $\mathrm{y}$ finalmente la combinación $\mathrm{R}$ y $\mathrm{Z}$ durante 2 meses diariamente. En pacientes VIH + consideran de elección tanto la $\mathrm{H}$ durante 9 meses como $\mathrm{R}$ con $\mathrm{Z}$ durante 2 meses y como alternativa $R$ durante 4 meses (21).

\section{INTERACCIONES}

Los dos medicamentos básicos del tratamiento de la TB, $\mathrm{H}$ y $\mathrm{R}$, presentan numerosas interacciones que deben de tenerse en cuenta sobre todo cuando el paciente necesita la administración de otros medicamentos con un estrecho margen terapéutico. Las interacciones se producen a nivel del sistema citocromo P-450 del hígado sobre el que actúan de forma distinta, la $\mathrm{H}$ es un inhibidor enzimático, provocando habitualmente un aumento de los niveles sanguíneos de los medicamentos que se metabolizan a ese nivel con el consiguiente riesgo de toxicidad, mientras que la $\mathrm{R}$ es un inductor enzimático que acelera el aclaramiento de aquellos fármacos pudiendo ocasionar niveles subterapéuticos de los mismos. En la tabla III se recogen las principales interacciones de los medicamentos antituberculosos y algunas acciones para minimizar sus efectos $(11,22,23)$.

TABLA III

INTERACCIO NES M ÁS DESTACADAS DE LOS M EDICAM ENTOS ANTITUBERCULOSOS

\begin{tabular}{|c|c|c|c|}
\hline M edicamento & Interacción con: & Efecto & Actuación \\
\hline Isoniacida (H) & Antiepilépticos & $\begin{array}{c}\text { 个 niveles antiepiléptico } \Rightarrow \uparrow \\
\text { toxicidad SNC, hepática }\end{array}$ & $\begin{array}{l}\text { Ajustar dosis según niveles } \\
\text { sanguíneos }\end{array}$ \\
\hline \multirow[t]{8}{*}{$\begin{array}{l}\text { (inhibidor metabolismo } \\
\text { hepático otros medicamentos) }\end{array}$} & Alcohol & $\begin{array}{l}\uparrow \text { riesgo hepatitis, } \downarrow \text { efecto } \mathrm{H} \\
\text { por } \uparrow \text { metabolismo }\end{array}$ & $\begin{array}{c}\text { Evitar ingesta alcohol, } \\
\text { vigilancia hepatotoxicidad }\end{array}$ \\
\hline & Antiácidos & $\downarrow$ concentración $\mathrm{H}$ & Administrar $\mathrm{H} 2$ horas antes \\
\hline & Anticoagulantes orales & $\uparrow$ efecto anticoagulante & Ajustar dosis anticoagulante \\
\hline & Corticosteroides & Puede $\downarrow$ concentración de $\mathrm{H}$ & Ajustar dosis $\mathrm{H}$ \\
\hline & Disulfiram & $\uparrow$ hepatotoxicidad & Evitar asociación \\
\hline & Teofilina & $\begin{array}{c}\text { 个 niveles teofilina después de } \\
\text { varias semanas de inicio } \\
\text { tratamiento } \mathrm{H}\end{array}$ & $\begin{array}{l}\text { M onitorización niveles } \\
\text { teofilina }\end{array}$ \\
\hline & Haloperidol, benzodiazepinas & $\uparrow$ toxicidad SNC por $\downarrow$ metabolismo & Ajuste dosis \\
\hline & O tros fármacos antituberculosos & $\uparrow$ toxicidad por $\downarrow$ metabolismo & Vigilar reacciones adversas \\
\hline
\end{tabular}


TABLA III (CONT.)

\begin{tabular}{|c|c|c|c|}
\hline M edicamento & Interacción con: & Efecto & Actuación \\
\hline Rifampicina (R) & Anticoagulantes orales & $\begin{array}{l}\downarrow \text { efecto anticoagulante efecto } \\
\text { por } \uparrow \text { metabolismo }\end{array}$ & Ajustar dosis anticoagulantes \\
\hline \multirow[t]{12}{*}{$\begin{array}{l}\text { (inductor metabolismo } \\
\text { hepático de otros medicamentos) }\end{array}$} & Anticonceptivos orales & $\begin{array}{l}\downarrow \text { efecto anticonceptivo efecto } \\
\text { por } \uparrow \text { metabolismo }\end{array}$ & Cambiar método anticonceptivo \\
\hline & Antiepilépticos & $\downarrow$ efecto por $\uparrow$ metabolismo & $\begin{array}{l}\text { Ajustar dosis según niveles } \\
\text { sanguíneos }\end{array}$ \\
\hline & Antiácidos & $\downarrow$ concentración $\mathrm{R}$ & Administrar $R 2$ horas antes \\
\hline & Analgésicos opiáceos & $\begin{array}{c}\downarrow \text { efecto analgésico por } \uparrow \\
\text { metabolismo. Peligro síndrome } \\
\text { abstinencia }\end{array}$ & $\begin{array}{c}\text { Ajustar dosis. } \\
\text { Importante con M etadona }\end{array}$ \\
\hline & Corticosteroides & $\downarrow$ efecto corticoides por $\uparrow$ metabolismo & Aumentar dosis entre 2 y 3 veces \\
\hline & Ciclosporina & $\downarrow$ efecto por $\uparrow$ metabolismo & M onitorización niveles plasmáticos \\
\hline & Digoxina & $\downarrow$ efecto digital por $\uparrow$ metabolismo & M onitorización niveles plasmáticos \\
\hline & Hipoglucemiantes & $\begin{array}{c}\downarrow \text { efecto hipoglucemiante por } \uparrow \\
\text { metabolismo }\end{array}$ & $\begin{array}{l}\text { Aumentar dosis. } \\
\text { Difícil control }\end{array}$ \\
\hline & Ketoconazol & $\begin{array}{l}\downarrow \text { efecto antifúngico y de la } \mathrm{R} \text { por } \uparrow \\
\text { metabolismo y disminución absorción } \mathrm{R}\end{array}$ & Separar su administración \\
\hline & $\begin{array}{c}\text { Antirretrovirales inhibidores } \\
\text { de la proteasa }\end{array}$ & $\downarrow$ efecto antiviral por $\uparrow$ metabolismo & $\begin{array}{l}\text { Evitar uso conjunto. } \\
\text { Valorar uso de rifabutina }\end{array}$ \\
\hline & $\begin{array}{l}\text { M edicamentos que actúan } \\
\text { sistema cardiovascular }\end{array}$ & $\begin{array}{c}\downarrow \text { efecto sistema cardiovascular } \\
\text { por } \uparrow \text { metabolismo }\end{array}$ & $\begin{array}{l}\text { Aumentar dosis en función } \\
\text { de respuesta }\end{array}$ \\
\hline & Teofilina & $\downarrow$ efecto teofilina por $\uparrow$ metabolismo & M onitorización niveles teofilina \\
\hline \multirow[t]{2}{*}{ Pirazinamida (Z) } & Alopurinol & $\uparrow$ concentración ácido úrico & Evitar empleo simultáneo \\
\hline & Protionamida & $\uparrow$ efectos secundarios Z & $\begin{array}{l}\text { Precaución, vigilar } \\
\text { reacciones adversas }\end{array}$ \\
\hline Etambutol (E) & M edicamentos neurotóxicos & $\begin{array}{l}\text { Potenciación mutua efectos adversos } \\
\text { (neuritis óptica y periférica) }\end{array}$ & Vigilar reacciones adversas \\
\hline \multirow{6}{*}{$\begin{array}{l}\text { Estreptomicina (S) y otros } \\
\text { aminoglucósicos }\end{array}$} & Anticoagulantes orales & $\uparrow$ efecto anticoagulante & Ajustar dosis anticoagulante \\
\hline & Antiinflamatorios no esteroideos & $\begin{array}{l}\text { } \text { efectos oto y nefrotóxicos por } \\
\text { aumento niveles aminoglucósidos }\end{array}$ & $\begin{array}{c}\text { Evitar utilización simultánea. } \\
\text { M ayor peligro en ancianos. } \\
\text { M onitorizar niveles y función renal }\end{array}$ \\
\hline & Capreomicina & $\begin{array}{l}\text { Tefectos oto y nefrotóxicos y } \\
\text { bloqueo neuromuscular }\end{array}$ & No utilización simultánea \\
\hline & $\begin{array}{c}\text { Diuréticos del asa } \\
\text { (furosemida, torasemida, } \\
\text { ac. etacrínico) }\end{array}$ & $\uparrow$ efectos oto y nefrotóxicos & $\begin{array}{c}\text { Evitar. Si no, ajustar mínima } \\
\text { dosis eficaz. } \\
\text { Pruebas función renal }\end{array}$ \\
\hline & Ciclosporina & 个efectos nefrotóxicos & $\begin{array}{c}\text { Evitar. Si no, ajustar mínima } \\
\text { dosis eficaz. } \\
\text { Pruebas función renal }\end{array}$ \\
\hline & $\begin{array}{l}\text { Cefalosporinas, clindamicina, } \\
\text { vancomicina }\end{array}$ & $\uparrow$ efectos nefrotóxicos & $\begin{array}{c}\text { Evitar. Si no, ajustar mínima } \\
\text { dosis eficaz. } \\
\text { Pruebas función renal }\end{array}$ \\
\hline \multirow[t]{5}{*}{ Quinolonas (FQ) } & Antiácidos & $\downarrow$ concentración FQ & Administrar R 2 horas antes \\
\hline & Anticoagulantes orales & $\uparrow$ efecto anticoagulante & Ajustar dosis anticoagulante \\
\hline & Fenitoína & $\begin{array}{c}\uparrow \text { niveles antiepilépticos } \\
\uparrow \text { toxicidad SNC } \downarrow \text { concentración FQ }\end{array}$ & $\begin{array}{c}\text { Ajustar dosis según niveles } \\
\text { sanguíneos }\end{array}$ \\
\hline & Sales de hierro, sales de zinc & $\downarrow$ concentración FQ & Evitar asociación \\
\hline & Teofilina & $\uparrow$ niveles teofilina & Ajustar dosis teofilina. M onitorizar \\
\hline
\end{tabular}




\section{REACCIONES ADVERSAS}

Los efectos adversos a los medicamentos antituberculosos pueden leves o bien poner riesgo la vida. Todos los pacientes a tratamiento deben de tener conocimiento acerca de los síntomas atribuibles a reacciones adversas graves así como a aque- llos cambios que resultan intrascendentes como son los cambios de pigmentación de los líquidos corporales cuando se utiliza R. En la tabla IV se resumen los principales efectos adversos de los medicamentos antituberculosos tanto de primera como de segunda línea $(11,22,23)$. En general ante síntomas menores la actuación sería:

TABLA IV

PRINCIPALES REACCIO NES ADVERSAS QUE PUEDEN PRESENTAR LOS M EDICAM ENTOS UTILIZADOS EN EL TRATAMIENTO DE LA TB

\begin{tabular}{ccc}
\hline M edicamento & Reacciones adversas & Signos y síntomas \\
\hline Isoniacida (Z) & Elevación enzimas hepáticas, & Náusea, vómito, dolor abdominal, \\
& hepatitis, neuropatía, efecto & abstemia, coluria, sensación \\
& sistema nervioso central & hormigueo en manos y pies
\end{tabular}

Actuación recomendada

Neuropatía más frecuente en alcohólicos, diabéticos y embarazada. Administrar vit. B6 25mg/ día. Riesgo hepatitis incrementado con la edad y consumo de alcohol. Ver interacciones. Control basal y periódicos de enzimas hepáticas

\begin{tabular}{|c|c|c|c|}
\hline Rifampicina (R) & $\begin{array}{l}\text { Hepatitis, trastorno GI, hemorragia, } \\
\text { efecto SNC, hipersensibilidad, } \\
\text { reacciones cutáneas. Pigmentación } \\
\text { naranja fluidos orgánicos. }\end{array}$ & $\begin{array}{l}\text { Náusea, vómito, dolor abdominal, } \\
\text { abstemia, cefalea, insomnio, } \\
\text { coluria, sintomas semejante } \\
\text { gripe (más frecuente en tto.intern.) }\end{array}$ & $\begin{array}{l}\text { Ver interacciones: numerosas } \\
\text { e importantes. } \\
\text { Control basal y periódicos de } \\
\text { enzimas hepáticas y plaquetas }\end{array}$ \\
\hline Pirazinamida (Z) & $\begin{array}{l}\text { Hepatitis, reacciones cutáneas, } \\
\text { trastorno Gl, eritema, hiperuricemia, } \\
\text { gota (raro) }\end{array}$ & M alestar estomacal, artralgias & $\begin{array}{l}\text { Tratar hiperuricemia sólo si el paciente } \\
\text { tiene síntomas. } \\
\text { Control basal y periódicos de enzimas } \\
\text { hepáticas y niveles de ácido úrico }\end{array}$ \\
\hline Etambutol (E) & $\begin{array}{l}\text { Neuritis óptica, hiperuricemia } \\
\text { (más raro que anterior). }\end{array}$ & $\begin{array}{c}\text { Alteración en la visión y percepción } \\
\text { color, artralgias }\end{array}$ & $\begin{array}{c}\text { No recomendado en niños, excepto en TB } \\
\text { resistente. } \\
\text { Pruebas visuales mensuales }\end{array}$ \\
\hline $\begin{array}{l}\text { Estreptomicina (S) } \\
\text { Amikacina } \\
\text { Kanamicina } \\
\end{array}$ & $\begin{array}{l}0 \text { totoxicidad, toxicidad renal, } \\
\text { reacciones cutáneas. } \\
\text { Bloqueo unión neuromuscular }\end{array}$ & $\begin{array}{l}\text { Hipoacusia, disfunción vestibulares. } \\
\text { Parestesias peribucales. M areo }\end{array}$ & $\begin{array}{l}\text { Evitar o reducir dosis en adultos }>60 \text { años. } \\
\text { Control basal y mensual audición y función renal }\end{array}$ \\
\hline Quinolonas (FQ) & $\begin{array}{c}\text { M alestar GI, alteraciones sistema } \\
\text { nervioso central, reacciones cutáneas, } \\
\text { fotosensiblidad }\end{array}$ & $\begin{array}{l}\text { Anorexia, náuseas, vómitos, mareos, } \\
\text { cefalea }\end{array}$ & $\begin{array}{l}\text { Evitar en niños y embarazadas } \\
\text { (riesgo de artropatía). Ver interacciones }\end{array}$ \\
\hline $\begin{array}{l}\text { Protionamida (Pt) } \\
\text { sist }\end{array}$ & $\begin{array}{l}\text { Hepatitis, malestar } \mathrm{GI} \text {, alteraciones } \\
\text { tema nervioso central, reacciones cutáneas, } \\
\text { hipoglucemia, neuropatía periférica }\end{array}$ & $\begin{array}{l}\text { Anorexia, náuseas, vómitos, mareos, cefalea, } \\
\text { reacciones psicóticas, depresión }\end{array}$ & $\begin{array}{l}\text { Mejor tolerancia al ingerir con zumo o leche, } \\
\text { al acostarse. Evitar en embarazo. } \\
\text { Precaución alcoholismo. } \\
\text { Control basal y periódicos de enzimas hepáticas, } \\
\text { glucemia, estado mental }\end{array}$ \\
\hline Capreomicina $(\mathrm{Cm})$ & $\begin{array}{l}\text { Similares a estreptomicina, hipopotasemia, } \\
\text { hipocalcemia y hipomagnesemia. }\end{array}$ & $\begin{array}{l}\text { Similares a estreptomicina, menor } \\
\text { riesgo ototoxicidad. }\end{array}$ & $\begin{array}{l}\text { No en embarazo y niños. } \\
\text { Control basal y mensual audición, } \\
\text { función renal, electrolitos en sangre }\end{array}$ \\
\hline $\begin{array}{c}\text { Ácido para- } \\
\text { aminosalicílico (PAS) }\end{array}$ & $\begin{array}{c}\text { Hepatitis, malestar GI, efecto } \\
\text { antitiroideo, hipersensibilidad, } \\
\text { hipopotasemia }\end{array}$ & $\begin{array}{l}\text { Anorexia, náuseas, molestias abdominales, } \\
\text { hipotiroidismo y bocio } \\
\text { (administración prologada). }\end{array}$ & $\begin{array}{c}\text { Evitar en IR, ICC. Precaución en hipertensos } \\
\text { si se emplea la sal sódica. } \\
\text { Disminuir dosis en intolerancia GI. } \\
\text { Control basal y periódicos de enzimas } \\
\text { hepáticas, electrolitos, tiroxina }\end{array}$ \\
\hline Cicloserina (Cs) & $\begin{array}{c}\text { Hepatitis, alteraciones sistema nervioso } \\
\text { central, trastornos del comportamiento, } \\
\text { hipersensiblidad }\end{array}$ & $\begin{array}{l}\text { Irritabilidad, cefalea, temblores, convulsiones, } \\
\text { letargia, insomnio, coma, tendencia al suicidio }\end{array}$ & $\begin{array}{c}\text { No utilizar en epilepsia, trastornos mentales o } \\
\text { alcoholismo. Precaución en IR. Vitamina B6 } \\
\text { puede disminuir efectos SNC. } \\
\text { Control basal y periódicos de enzimas hepáticas, } \\
\text { neurológicos y estado mental }\end{array}$ \\
\hline Rifabutina & $\begin{array}{l}\text { Hepatitis, uveítis, malestar GI, } \\
\text { persensibilid, neutropenia, trombocitopenia, } \\
\text { artralgia, hiperpigmentación piel. }\end{array}$ & Anorexia, náuseas, vómitos. & $\begin{array}{c}\text { Reducir dosis en IR. No niños. Ver interacciones. } \\
\text { Control basal y periódicos de pruebas hematológicas, } \\
\text { de enzimas hepáticas y oftalmológicas }\end{array}$ \\
\hline
\end{tabular}


Intolerancia digestiva: se puede mejorar fraccionando la dosis los primeros días, ingiriendo el fármaco después del desayuno o administrándolo al acostarse.

Hiperuricemia:

-Sin molestias: no realizar cambios.

-Artralgia moderada: control con antiinflamatorios.

-Artralgia severa o crisis gotosa: suspender fármaco causante 8 días y reanudar tratamiento después, si regresa síntoma, cambiar pauta tratamiento.

Reacción cutánea:

- Si moderada y no cede con antihistamínicos suspender 8 días tratamiento y reintroducirla gradualmente, si reaparece sustituir medicamento causante.

- Si reacción severa que no cede con antihistamínicos, suprimir el tratamiento, identificar el fármaco causante y cambiarlo definitivamente por otro.

En caso de presentarse algún efecto tóxico grave como hepatitis, alteraciones hematológicas o neuropatía el tratamiento deberá suspenderse de inmediato. En la figura 1 se recoge la actuación que debe realizarse ante una elevación las enzimas hepáticas durante un tratamiento convencional de 6 meses (24).

\section{CONTROL DEL CUMPLIMIENTO Y TRATAMIENTO DIRECTAMENTE OBSERVADO (TDO)}

Se ha estimado que es usual que entre un $20-50 \%$ de los pacientes tomen de forma incorrecta o no completan el régimen terapéutico prescrito. El riesgo de incumplimiento es mayor después de la fase inicial del mismo, transcurridas las primeras 6-8 semanas. Los fallos en la cumplimentación del tratamiento conllevan a fracasos terapéuticos y aparición de resistencias secundarias. Dada la dificultad de prever el cumplimiento por parte del paciente se han desarrollado distintas técnicas para poder evaluarlo. Las técnicas indirectas consistentes en recuento de comprimidos sobrantes, interrogatorios, etc. no aseguran la ingesta del tratamiento. Existen métodos directos que permiten la determinación de los niveles de los medicamentos en sangre o sus metabolitos en la orina, pero son difíciles de incluir en un programa de control rutinario. Sin embargo, existen métodos cualitativos sencillos de detección de $\mathrm{H}$ y metabolitos en orina $(25,26)$.

Los métodos anteriormente citados tampoco aseguran un completo cumplimiento. La única certeza de adhesión es mediante el llamado tratamiento directamente observado (TDO) que consiste en observar cómo el enfermo toma la medicación, controlando si la ha ingerido. Su eficacia ha hecho que se le considere actualmente el estándar del tratamiento antituberculoso y su implantación sea recomendada por la OMS $(14,27)$, su instauración en España es poco común $(4,5)$. Su uso, en todo caso, es especialmente recomendable cuando el incumplimiento es previsible (alcoholismo, indigencia, prisión, desestructuración familiar o social, toxicómanos, trastornos mentales, antecedentes de abandono del tratamiento, prostitución, inmigrantes o retratamientos) y en algunos casos de tratamiento de TB latente. Debería ser obligatorio en incumplidores en los que se ha intentado otras medidas de cumplimiento (amplia información, apoyo familiar, incentivos, entrega gratuita de la medicación, etc.).

En los pacientes más necesitados se puede favorecer el TDO en plazas de internamiento, habitualmente en centros sociosanitarios con estancias ilimitadas o mediante equipos ambulatorios que administran la medicación allí donde el paciente está (domicilio, pensión, la propia calle, etc.). El TDO también puede aplicarse en centros de atención primaria, dispensarios antituberculosos, programas de mantenimiento con metadona (muy útiles en UDVP), centros para alcohólicos y/o indigentes, etc. Se recomienda la creación de este tipo de centros y de equipos en aquellas zonas donde sean necesarios (4).

También puede ser útil el tratamiento supervisado semanal (TSS) y el TDO intermitente desde el inicio (dos veces/semana), que operativamente es más fácil. Si el tratamiento no es aceptado o se abandona, debe ofertarse de forma voluntaria el internamiento $\mathrm{y}$, en bacilíferos que rechacen esta medida, debería procederse al TDO obligatorio o a ingreso por orden judicial si fuera preciso. Los enfermos suelen responder bien a incentivos. Los más importantes son la metadona en los toxicómanos, el ingreso de indigentes en centros sanitarios, la comida, la vivienda y el transporte. En algunos pacientes bacilíferos que precisen TDO es útil el ingreso hospitalario durante 3 semanas, seguido del traslado a un centro sociosanitario hasta cumplir la fase intensiva del segundo mes de tratamiento (como mínimo) y después TDO ambulatorio. Si no se dispone de estos recursos el TSS constituye una alternativa (4). Se considera que estos programas son coste efectivos (28).

\section{TRATAMIENTO EN SITUACIONES ESPECIALES}

\section{INMUNODEFICIENCIAS}

Los pacientes con insuficiencia renal crónica, desnutrición, tumores de origen hemático o reticuloendotelial, e infección por HIV, así como aquellos que reciben medicamentos inmunosupresores tienen mayor riesgo de presentar TB en caso de estar infectado. Si se desarrolla la enfermedad tienen mayor riesgo de TB extrapulmonar y diseminada. En general el tratamiento de la TB extrapulmonar es similar a la TB pulmonar (13,29-34).

Según el Consenso (4) los enfermos infectados por VIH deben ser tratados por un especialista y deben tenerse en cuenta las numerosas interacciones farmacocinéticas que existen entre las rifamicinas y los inhibidores de la proteasa (IP) y los inhibidores no nucleósicos de la trascriptasa inversa. Debe usarse TDO para asegurar el cumplimiento del tratamiento (de la tuberculosis y del VIH) en UDVP y en otros pacientes con previsible mal cumplimiento. Deben recomendarse pautas de 9 meses ya que se han observado más recaídas en pautas de sólo 6 meses. También debe priorizarse el uso de fármacos combinados para evitar monoterapias. El Consenso propone:

1. En pacientes sin tratamiento antirretroviral, prescribir una pauta de 9 meses (dos con $\mathrm{H}, \mathrm{R}$ y Z más siete $\mathrm{H}$ y R) y retrasar el inicio de tratamiento antirretroviral hasta pasado el segundo mes. A partir del tercer mes, se puede optar por: a) sustituir la $\mathrm{R}$ por rifabutina a dosis de $150 \mathrm{mg} /$ día (H más rifabutina hasta completar 9 meses) y utilizar como IP nelfinavir ( $750 \mathrm{mg} / 8 \mathrm{~h}$ ) o indinavir (subir dosis a $1.000 \mathrm{mg} / 8 \mathrm{~h}$ ) o mantener la dosis de rifabutina de $300 \mathrm{mg} /$ día y utilizar nevirapina (400 mg/día), y b) mantener $\mathrm{H}$ y $\mathrm{R}$ hasta completar 9 meses y seleccionar ritonavir como inhibidor de proteasa o efavirenz como inhibidor no nucleósido de la transcriptasa inversa. En ambas situaciones deben monitorizarse la carga viral y los CD4.

2. En pacientes que ya reciben un tratamiento antirretroviral con el que se consigue controlar la infección por VIH, no 
se recomienda interrumpirlo, por lo que se realizarán los ajustes necesarios para adecuar los tratamientos a los esquemas enunciados en a) o b). La $\mathrm{R}$ y la nevirapina son inductores del citocromo P-450 y en los UDVP interaccionan con la metadona acelerando su metabolismo, por lo que debe incrementarse la dosis de metadona. No existen datos con efavirenz, aunque también es un inductor del citocromo P-450.

Otra situación de inmunodeficiencia son los pacientes inmunodeprimidos por el tratamiento inmunosupresor utilizado después de un transplante de órgano. Habitualmente responden adecuadamente a la quimioterapia antituberculosa convencional. Si se emplea ciclosporina como agente inmunosupresor, éste interactúa con el metabolismo de la R (Tabla III) . Se producen niveles más bajos de ciclosporina y por tanto se incrementa el rechazo de trasplante. El tratamiento intermitente es difícil de aplicar cuando se intentan controlar los niveles plasmáticos de ambos fármacos. Al igual que con los IP, la rifabutina tiene un menor efecto sobre la ciclosporina que la $\mathrm{R}$. El régimen de administración diario conteniendo rifabutina puede resultar idóneo para tratar la TB en estos pacientes. El control de los niveles plasmáticos ayuda a optimizar el tratamiento farmacológico (13).

\section{EMBARAZO Y LACTANCIA}

El riesgo de una TB no tratada en una mujer embarazada es mayor para ella y su feto que el tratamiento. Los cuatro fármacos de primera línea de elección (R, H, E y Z) se consideran seguros y no se han asociado a malformaciones (35), por lo que debe utilizarse el tratamiento convencional. Las pautas, el control y seguimiento ha de ser el habitual. Se aconseja la adición de piridoxina a todas las embarazadas que ingieran $\mathrm{H}$. No debe utilizarse $\mathrm{S}$ ni otro aminoglucósido por el riesgo de ototoxicidad (hipoacusia) que pueden producir en el feto. Como medicamento de segunda línea se considera que ciprofloxacino presenta el mejor perfil de seguridad.

Los niños recién nacidos de madre con una tuberculosis activa deben recibir tratamiento para una tuberculosis latente con H. Se evitará en estos casos la lactancia materna puesto que la madre estará también tomando dicho medicamento como parte de su tratamiento. En caso de no estar activa la enfermedad en el momento del parto la mujer puede iniciar la lactancia, se excretan cantidades subterapéuticas en la leche. Algunos autores recomienda amamantar al niño antes de la toma del tratamiento y sustituir la primera toma después éste por un lactancia artificial con el fin de evitar altos niveles séri$\cos (36)$.

\section{INSUFICIENCIA RENAL}

La OMS establece que en fallo renal puede utilizarse la $\mathrm{H}$, $\mathrm{R}$ y $\mathrm{Z}$ a dosis normales y recomienda como pauta más segura la de 2 meses de la triple asociación de los medicamentos antes citados y 6 meses de R e H (14). Sin embargo, si el aclaramiento de creatinina baja de $10 \mathrm{ml} / \mathrm{min}$ se aconseja reducir la dosis diaria en un $50 \%$.

Deben evitarse fármacos nefrotóxicos o los que se eliminen por vía renal, como son la estreptomicina, kanamicina, capreomicina, etambutol, cicloserina y fluorquinolonas. Si fuera necesaria su utilización deben ajustarse las dosis e inter- valos en función de los niveles séricos determinados. Los medicamentos antituberculosos son aclarados de forma variable por hemodiálisis. En los días de diálisis la toma del tratamiento debe hacerse habitualmente después de la diálisis $(11,13)$.

\section{ENFERMEDAD HEPÁTICA}

En general los pacientes con enfermedad hepática crónica (hepatitis crónica activa o cirrosis, portadores crónicos del virus de la hepatitis $\mathrm{B}$ y C) no necesitan un ajuste posológico de los fármacos antituberculosos (12). No obstante, es necesario hacer una vigilancia más frecuente en los primeros meses del tratamiento, realizando controles de la función hepática semanales durantes las dos primeras semanas y posteriormente cada 2 semanas (37).

Si durante el tratamiento se observa una elevación de las transaminasas a 5 veces los valores normales se debe reevaluar el tratamiento siguiendo el protocolo de la figura 1.

En caso de hepatopatía grave o descompensada se debe evitar en los medicamentos de primera línea la $\mathrm{Z}$ y en los de segunda protionamida por ser hepatotóxicos. Las pautas recomendadas de mayor a menor hepatotoxicidad son: 2HRE/7HR, 2HES/16HE o 2RES/10RE y 6ESCs/18ECs, en esta última se puede sustituir la cicloserina $(\mathrm{Cs})$ por capreomicina $(\mathrm{Cm})(12,14)$.

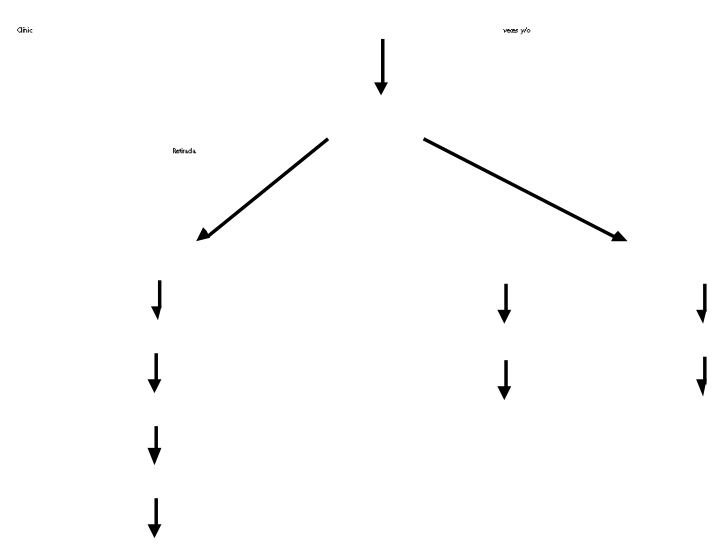

Fig. 1. Protocolo de actuación ante la sospecha de hepatotoxidad del tratamiento habitual de la tuberculosis.

\section{PROBLEMÁTICA DE LAS MULTIRRESISTENCIAS}

En general, en los casos de fracaso terapéutico o de recidiva, tras el empleo de un régimen estandar, es muy probable que aparezca una resistencia adquirida a la $\mathrm{H}$ y a la $\mathrm{R}$. Estos casos, como se mencionó anteriormente, deben ser derivados a unidades especializadas. En espera de los resultados de las pruebas de sensibilidad, no se debe prescribir un régimen que incluya a ambos fármacos. Los regímenes elegidos deberán consistir en una combinación de medicamentos básicos ( $\mathrm{S}, \mathrm{Z}$ y E) y medi- 
camentos de segunda línea. No deben de utilizarse para tratar una TB multiresistente los derivados de la $\mathrm{R}$ como la rifabutina, especialmente cuando hay resistencia a $\mathrm{R}$, la clofazimina que es poco activa contra Mycobacterium tuberculosis. En la tabla II se recogen las dosificaciones recomendadas, los fármacos disponibles en España y el coste del tratamiento/día en precio venta al público (euros) en envase normal (38).

Los esquemas de tratamiento se basan en la sensibilidad y riesgo de toxicidad. Siempre que exista una resistencia a $R$ los tratamientos serán de una duración mínima de 18 meses. La OMS (39) propone mientras no se dispongan de resultados de las pruebas de sensibilidad un "régimen tercera línea" que comprenda: al menos 3 medicamentos no utilizados hasta entonces: kanamicina (o amikacina), etionamida (no está disponible en España, se está importando al protionamida que tiene una estructura y actividad similar), ofloxacino (en España hay actualmente comercializadas otras quinolonas con igual o mayor eficacia: ciprofloxacino, levofloxacino) más pirazinamida (Z). Tras la conversión bacteriológica (generalmente a los 3 ó 4 meses), si no se dispone de los resultados de la prueba inicial de sensibilidad, se procederá a una fase de continuación de 18 meses administrando al menos los 2 medicamentos mejor tolerados y de ordinario más activos: etionamida (protionamida) y ofloxacino (levofloxacino). Recomiendan valorar el tratamiento quirúrgico en casos de resistencias a 4 fármacos. En la tabla $\mathrm{V}$ se recogen algunas de las pautas posibles en función de las los resultados de las resistencias detectadas (39-42).

\section{PERSPECTIVAS DE TRATAMIENTO}

Dentro del campo de la inmunoterapia están en estudio más de 100 vacunas animales de distintos tipos ensayadas en modelos. Unas son subunidades antigénicas proteicas, lipídicas o de carbohidratos de $M$. tuberculosis en varias formulaciones, que son potencialmente específicas, definidas y seguras, pero como desventaja presentan una limitada respuesta inmunológica. Otro tipo de vacunas consisten en ADN que codifican varios antígenos de M. tuberculosis, son fáciles de producir, relativamente económicas e inducen respuestas inmunes duraderas mediadas por células. Por último vacunas vivas atenuadas incluyendo especies micobacterianas no patógenas y BCG genéticamente manipulada que expresan antígenos inmunodominantes de $M$. tuberculosis, la ventaja de éstas es la amplitud de rango antigénico y persistencia de la respuesta pero su seguridad en inmunodeficientes no ha sido establecida $(43,44)$.

La lucha farmacológica contra la TB se está desarrollando en distintos campos. Se están buscando sistemas que mejoren el cumplimiento terapéutico mediante el desarrollo de formas far-
TABLA V

\begin{tabular}{|c|c|c|}
\hline \multicolumn{3}{|c|}{$\begin{array}{c}\text { PAUTAS POSIBLES EN PARA EL TRATAM IENTO DE } \\
\text { TUBERCULOSIS M ULTIRRESISTENTE. } \\
\text { DURACIÓN M ÍNIM A EN M ESES }\end{array}$} \\
\hline Resistencia & Fase inicial & $\begin{array}{l}\text { Fase continuación (después } \\
\text { negativización cultivo) }\end{array}$ \\
\hline H (S) & $\begin{array}{c}2-3 \text { meses } \\
\text { Rtaminoglucósido* }+Z+E\end{array}$ & $\begin{array}{c}6 \text { meses } \\
R+E\end{array}$ \\
\hline$H, E(S)$ & $\begin{array}{l}\text { 2-3 meses } \\
\text { R+aminoglucósido* }+Z+ \\
\text { etionamida** }\end{array}$ & $\begin{array}{c}6 \text { meses } \\
\text { R+ etionamida** }\end{array}$ \\
\hline$H, Z(S)$ & $\begin{array}{l}\text { 2-3 meses } \\
\text { R+aminoglucósido* }+E+ \\
\text { etionamida** }\end{array}$ & $\begin{array}{c}6 \text { meses } \\
\mathrm{R}+\text { etionamida** }\end{array}$ \\
\hline$H, R(S)$ & $\begin{array}{l}3 \text { meses } \\
\text { aminoglucósido* }+\mathrm{Z}+\mathrm{E} \\
\text { etionamida**+FQ }\end{array}$ & $\begin{array}{c}18 \text { meses } \\
\text { E+ etionamida** }+F Q\end{array}$ \\
\hline$H, R, E(S)$ & $\begin{array}{l}3 \text { meses } \\
\text { aminoglucósido* }+\mathrm{Z}+\mathrm{FQ}+ \\
\text { etionamida**+Cs/PAS }\end{array}$ & $\begin{array}{c}18-24 \text { meses } \\
\text { etionamida** }+\mathrm{FQ}+\mathrm{Cs} / \mathrm{PAS}\end{array}$ \\
\hline$H, R, Z(S)$ & $\begin{array}{l}3 \text { meses } \\
\text { aminoglucósido*+E+FQ+ } \\
\text { etionamida**+Cs/PAS }\end{array}$ & $\begin{array}{c}18-24 \text { meses } \\
\text { etionamida** }+F Q+E / C s / P A S\end{array}$ \\
\hline$H, R, E, Z(S)$ & $\begin{array}{l}3 \text { meses } \\
\text { aminoglucósido* }+\mathrm{FQ}+ \\
\text { etionamida** }+ \text { CS }+ \text { PAS*** }\end{array}$ & $\begin{array}{c}18-24 \text { meses } \\
\text { etionamida** }+F Q+C s / P A S\end{array}$ \\
\hline
\end{tabular}

$\mathrm{H}=$ isoniazida, $\mathrm{R}=$ rifampicina, $\mathrm{Z}=$ pirazinamida, $\mathrm{E}=$ etambutol, $\mathrm{S}=$ estreptomicina, FQ = Fluorquinolona (ciprofloxacino, levofloxacino), PAS $=$ Ac. paraaminosalicílico,$C s=$ Cicloserina .

* Si resistencia a aminoglucósidos sustituir por capreomicina.

**Sustituir por protionamida en España o una FQ las 3 primeras pautas.

***Si intolerancia a alguno se podría sustituir por clofazimina, amoxicilina-ác. clavulánico.

macéuticas de acción sostenida o implantables de medicamentos ya empleados o nuevos y de medicamentos que acorten el tratamiento y permitan la administración intermitente (11). Por otro lado se están buscando nuevos mecanismos de acción para poder combatir las resistencias. En modelos animales se están probando nuevas moléculas oxazolidinonas (45) que inhiben la síntesis proteica en fases tempranas, entre ellas se encuentra el linelozid (46). Otro grupo es el de los el nitroimidazopiranos, con una estructura similar al metronidazol (43). En la actualidad, con el conocimiento de la secuencia del genoma de $M$. tubercu losis se está probablemente en mejor posición para el desarrollo de nuevos medicamentos.

\section{Bibliografía}

1. World Health Organization. WHO report 2001. Global Tuberculosis Control. WHO/CDS/TB/2001.287. Ginebra: World Health Organization; 2001.

2. Salgueiro Rodriguez M, Zamarrón Sanz C, Alvarez-Calderon Prat P, Tumbeiro Novoa M, Penela Penela P, Valle Vazquez JM, et al. Estudio epidemiológico de la tuberculosis en el área sanitaria de Santiago de Compostela durante los años 1989, 1990 y 1991. An Med Interna (Madrid) 1993; 10: 427-432.

3. Salgueiro Rodriguez M, Zamarrón C, Otero Y, Álvarez-Dobaño JM,

Arias MR, Álvarez-Calderón P, et al. Estudio epidemiológico de la tuberculosis en el área sanitaria de Santiago de Compostela durante los años 1992,1993 y 1994. An Med Interna (Madrid) 1996; 13: 111-114.

4. Conferencia de consenso. Documento de consenso sobre la prevención y control de la tuberculosis en España. Med Clin (Barc) 1999; 113: 710715 .

5. Grupo de Trabajo del PMIT. Diagnóstico y tratamiento de la tuberculosis en España: resultados del Proyecto Multicéntrico de Investigación en Tuberculosis (PMIT). Med Clin (Barc) 2001: 116: 167-173. 
6. Salgueiro Rodriguez M, Zamarrón C, Gonzalez Barcala J, Vilas Iglesias A, Suarez Antelo J, Duran Rivas JL, et al. Estudio epidemiológico de la tuberculosis en el área sanitaria de Santiago de Compostela durante los años 1995,1996, 1997 y 1998. An Med Interna (Madrid) 2001; 18: 20-23.

7. Organización Mundial de la Salud. Modelo de Información sobre Prescripción de Medicamentos. Medicamentos utilizados en la enfermedades micobacterianas. Ginebra: Organización Mundial de la Salud; 1992.

8. World Health Organization. TB/VIH: A clinical manual. Ginebra: WHO, 1996.

9. Alzeer AH, FitzGerald JM. Corticosteroids and tuberculosis: risks and use as adjunct therapy. Tubercle Lung Dis 1993; 74: 6-11.

10. Grupo de Trabajo sobre Tuberculosis. Consenso Nacional para el Control de la Tuberculosis en España. Med Clin (Barc) 1992; 98: 24-31.

11. Fujiwara PI, Simone PM, Munsiff SS. Treatment of Tuberculosis. En: Tuberculosis. A Comprehensive International Approach, ed. Reachman LB, Hershfield ES, Marcel Dekker, Inc, New York, 2000; 401-446.

12. Ruiz Manzano J, Andreo F, Prats M. Tratamiento: estrategias futuras. En: Sauret Valet J . Tuberculosis. Visión actual. Aula Médica Ediciones, Madrid, 2001; 165-191.

13. Patterson PE, Kimerling ME, Bailey WC, Dunlap NE. Quimioterapia de la tuberculosis. En: Schlossberg D, editor.Tuberculosis e infecciones por micobacterias no tuberculosas. 4 ed. México: McGrawHill; 2000. p.79-94.

14. World Health Organization. Treatment of tuberculosis: Guidelines for national programmes. Second Edition 1997 WHO/TB/97.220 World Health Organization, 1997.

15. Mwandumba HC, Squire SB. Fully intermittent dosing with drugs for tuberculosis. The Crochane Library, Issue 2, Oxford, 2001.

16. Gómez Rodriguez F, Bernal Bermúdez JA, Garcia Egido, A. Evaluación y tratamiento de la tuberculosis latente en el adulto. Med Clin (Barc) 2001; 117: 111-114.

17. Cohn DL, O'Brien RL. Targeted tuberculin testing and treatment of latent tuberculosis infection. MMWR. Morb Mortal Wkly Rep 2000;4 9: $1-61$.

18. Gordin F, Chaisson RE, Matts JP, Miller C, Lourdes Garcia M, Hafner $\mathrm{R}$ et al. Rifampicin and pyrazinamide vs isoniazid for prevention of tuberculosis in HIV-infected persons; an international randomized trial. JAMA 2000; 283: 1445-50.

19. McCarthy M. US guidelines for treatment of latent tuberculosis. Lancet $2001 ; 358-816$.

20. Martínez Alfaro EM, Cuadra F, Solera J, Macia MA, Geijo P, Sanchez Martínez PA et al. Evaluacion de regimenes de quimioprofilaxis en pacientes infectados con virus de inmunodeficencia humana. Med Clin (Barc) 2000; 115: 161-5.

21. American Thoracic Society. Targeted tuberculin testing and testing and treatment of latent tuberculosis infection. Am J Respir Crit Care Med 2000; 161(S): S221-S247.

22. Villa C, Sánchez Quejido M, Aparicio Marban M, Iglesias J et al Tuberculosis. Panorama Actual Med 2000; 24: 543-561.

23. Rizack A. Compendio de interacciones adversas de medicamentos. The Medical Letter. Ed. J.R.Prous; 1999.

24. Sánchez Hernández IM, Ussetti Gil P, Melero Moreno C, Rey Duran R. Protocolo terapéutico de la tuberculosis en pacientes no inmunocomprometidos. Criterios de seguimiento y valoración de la toxicidad. Medicine 1998; 7: 3701-6.

25. Gavira R, Gomez F, Otero MJ, Barrueco M, Dominguez-Gil A. Seguimiento del tratamiento antituberculoso. Rev Clin Esp 1994; 194:677685.

26. García Ramos R, Túñez Bastida, V. Cumplimiento terapéutico en tuberculosis. Comparación de dos métodos. Farm Clín 1993; 10: 885-6.
27. World Health Organization.TB. A crossroads. WHO Report on the Global Tuberculosis Epidemic 1998: Ginebra: WHO, 1999.

28. Volmink J, Garner P. Interventions for promoting adherence to tuberculosis management. The Cochrane Library, Issue 2, Oxford, 2001.

29. Martínez Vázquez JM, Cabarcos Ortíz de Barrón A, Barrio Gómez E. Tuberculosis e infección por VIH: patogenia (Segunda de tres partes). An Med Interna (Madrid) 1997; 14: 310-316.

30. Lado Lado FL, Barrio Gómez E, Cabarcos Ortíz de Barrón A, Carballo Arceo E, Sánchez Leira J, Pérez del Molino ML, Antúnez López JR. Tuberculosis e infección por el virus de la inmunodeficiencia humana. Presentación clínica y diagnóstico. An Med Interna (Madrid) 1998; 15: 415-420.

31. Lado Lado FL, Barrio Gómez E, Carballo Arceo E, Cabarcos Ortíz de Barrón A. Clinical presentation of tuberculosis and the degree of inmunodeficiency in patients with HIV infection. Scand J Infect Dis 1999; 31:387-391.

32. Lado Lado FL, Barrio Gómez E, Carballo Arceo E, Cabarcos Ortíz de Barrón A. Tuberculosis e infección por el virus de la inmunodeficiencia humana: manifestaciones clínicas y rendimiento de procedimientos diagnósticos según las distintas formas de localización de la enfermedad.An Med Interna (Madrid) 2000;17:13-18.

33. Cabarcos Ortíz de Barrón A, Barrio Gómez E, Lado Lado FL, Rodríguez López I, Lorenzo Zúñiga V. Correlación entre el mantoux y la fracción tuberculínica de la prueba de la hipersensibilidad retardada, en una cohorte de pacientes VIH+, en función de su nivel de inmunodeficiencia, e incidencia de tuberculosis activa. An Med Interna (Madrid) 2000;17:632-636.

34. Lado Lado FL, Tuñez Bastida V, Golpe Gómez AL, Ferreiro Regueiro MJ, Cabarcos Ortíz de Barrón A. Tuberculosis extrapulmonar en nuestro medio. Formas de presentación. An Med Interna (Madrid) 2000; 17 : 637-641.

35. Bothamley G. Drug treatment for tuberculosis during pregnancy: safety consideration. Drug Saf 2001; 24 (7): 553-565.

36. Hamadech M, Glassroth J. Tuberculosis and pregnacy. Chest 1992;101:1114-1120.

37. Ormerod LP, Skinner C, Wales JM. Hepatoxicity fo antituberculosis drugs. Thorax 1996; 1996: 111-113.

38. Vila F. Medimecum. Guía de terapia farmacológica.2001. $6^{\circ}$ ed Adis Intenational Ldt; 2001

39. Crofton J, Chaulet P, Maher D. Directrices para el tratamiento de la tuberculosis farmacorresistente. WHO/TB/96.210 (Rev.1). Ginebra: Organización Mundial de la Salud, 1997.

40. Telenti A, Iseman M. Drug-resistant tuberculosis. What do we do now? Drugs 2000; 59: 171-179.

41. Telsak EE, Sepkowitz. Tratamiento de la tuberculosis resistente a múltiples fármacos. En: Tuberculosis e infecciones por micobacterias no tuberculosas. En: Schlossberg D, editor. Tuberculosis e infecciones por micobacterias no tuberculosas. 4 ed. México: McGrawHill; 2000. p. $95-$ 106.

42. Vidal Pla R. Tuberculosis multirresistente. En: Sauret Valet J . Tuberculosis. Visión actual. Aula Médica Ediciones, Madrid, 2001; 139-163.

43. Letvin NL, Bloom BR, Hoffman SL. Prospects for vaccines to protect against AIDS, tuberculosis, and malaria. JAMA 2001; 285: 606-611.

44. Friedland J. New vaccines for mycobacterial infections 'desperately needed'. Lancet 2001; 357: 203-6.

45. Mullan Z. New class of drugs provides hope for future of tuberculosis treatment. Lancet 2001; 355: 2223.

46. Wallace RJ, Brown-Elliot BA, Ward SC, Crist CJ, Mann LB, Wilson RW. Activities of Linezolid against rapidly growing Mycobacteria. Antimicrob Agents Chemother 2001; 45:764-767. 